\title{
Exercise-associated hyponatremia
}

\author{
William G. Schucany, MD
}

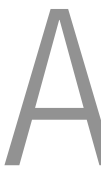

39-year-old white woman was brought to the emergency department at Baylor University Medical Center by emergency medical services. Prior to the ambulance arrival, she had complained of a progressively worsening headache, with the onset of nausea and vomiting, lethargy, and eventual obtundation. By the time the ambulance arrived on the scene, the patient was disoriented, unresponsive to verbal interaction, and responsive only to pain stimuli. Her vital signs were as follows: heart rate, 72 beats per minute; respiratory rate, 18 breaths per minute; blood pressure, 120/69 mm Hg; and temperature, $99^{\circ} \mathrm{F}$. En route to the emergency department, the patient received intravenous fluids, approximately $1 \mathrm{~L}$ of normal saline.

The patient was accompanied by her husband, who provided the history. He indicated that she was previously in good health, with no preexisting illnesses other than migraine headaches. The patient's husband had met his wife at the gym around noon. She had already completed a 2-hour workout, including tennis and weightlifting. The tennis game was outdoors on a humid $100^{\circ} \mathrm{F}$ Texas summer day. She had not eaten breakfast that day but was diligent about hydration, keeping a $1 \mathrm{~L}$ water bottle with her during the workout. She estimated that she had drunk $4 \mathrm{~L}$ of water before and during exercise. Following the tennis game, she continued her workout indoors in the weight room. There she developed a headache. Assuming that the headache was related to dehydration and/or heat, especially since she had not urinated, the patient continued drinking water and completed the weight-training workout. Afterwards, her headache worsened. She assumed that because of the severity, the headache was a migraine. She drove her own car home, followed by her husband. Once she got home, her headache continued to worsen and she felt something was seriously wrong. She quickly became
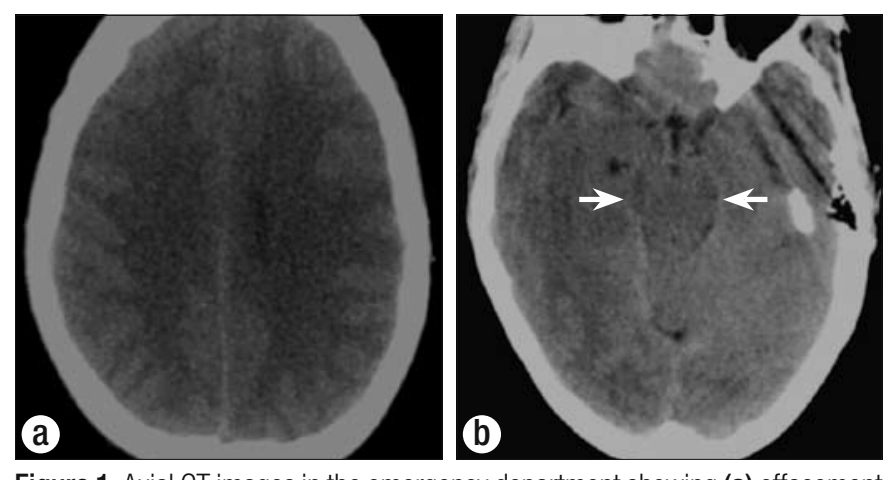

Figure 1. Axial CT images in the emergency department showing (a) effacement of the cortical sulci and (b) effacement of the basilar cisterns (arrows). nauseated and began vomiting. She then asked her husband to call an ambulance and within a few minutes became disoriented and unresponsive to verbal interaction. By the time the ambulance arrived, she was responsive only to pain stimuli.

In the emergency department, a number of laboratory tests were

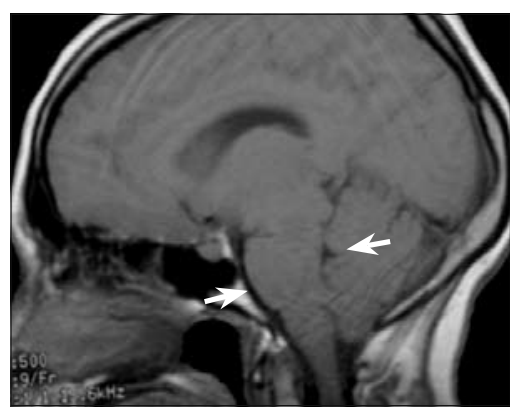

Figure 2. MR image in the emergency department. Sagittal T1-weighted image shows effacement of the prepontine cistern and cortical sulci and the decreased size of the fourth ventricle (arrows). done. Results were as follows: sodium, $123 \mathrm{mEq} / \mathrm{L}$; chloride, 95 $\mathrm{mEq} / \mathrm{L}$; potassium, $3.9 \mathrm{mEq} / \mathrm{L}$; calcium, $6.6 \mathrm{mg} / \mathrm{dL}$; creatine phosphokinase, $223 \mathrm{U} / \mathrm{L}$; hematocrit, 26.4\%; and hemoglobin, $9.9 \mathrm{~g} / \mathrm{dL}$. Urinalysis revealed an elevated urinary sodium of $190 \mathrm{mEq} / \mathrm{L}$ (reference range, 30-90). In addition, computed tomography (CT) scans and magnetic resonance (MR) images were obtained (Figures 1-3).

What is the most likely diagnosis?
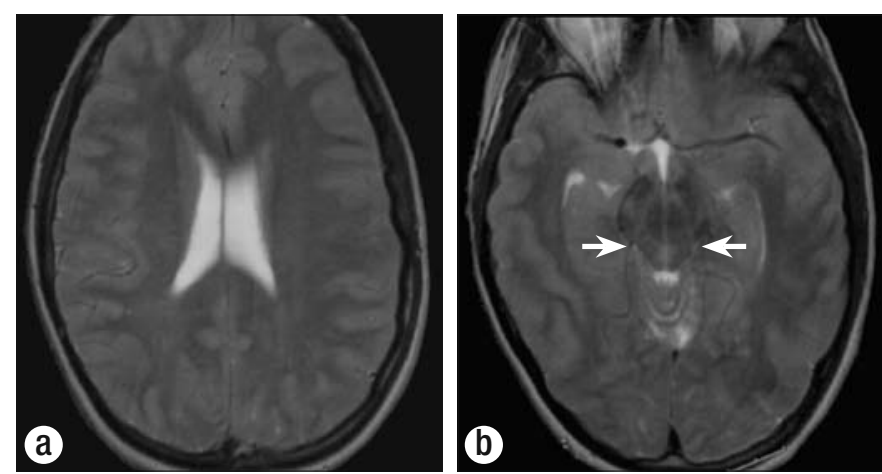

Figure 3. (a) Axial T2-weighted MR image shows effacement of the cortical sulci and generalized mild hyperintensity of the cortex. (b) Axial T2-weighted image shows effacement of the basilar cisterns (arrows), generalized mild hyperintensity of the cortex, and effacement of the cortical sulci.

From the Department of Radiology, Baylor University Medical Center, Dallas, Texas.

Corresponding author: William G. Schucany, MD, Department of Radiology, Baylor University Medical Center, 3500 Gaston Avenue, Dallas, Texas 75246 (e-mail: gschucany@americanrad.com). 

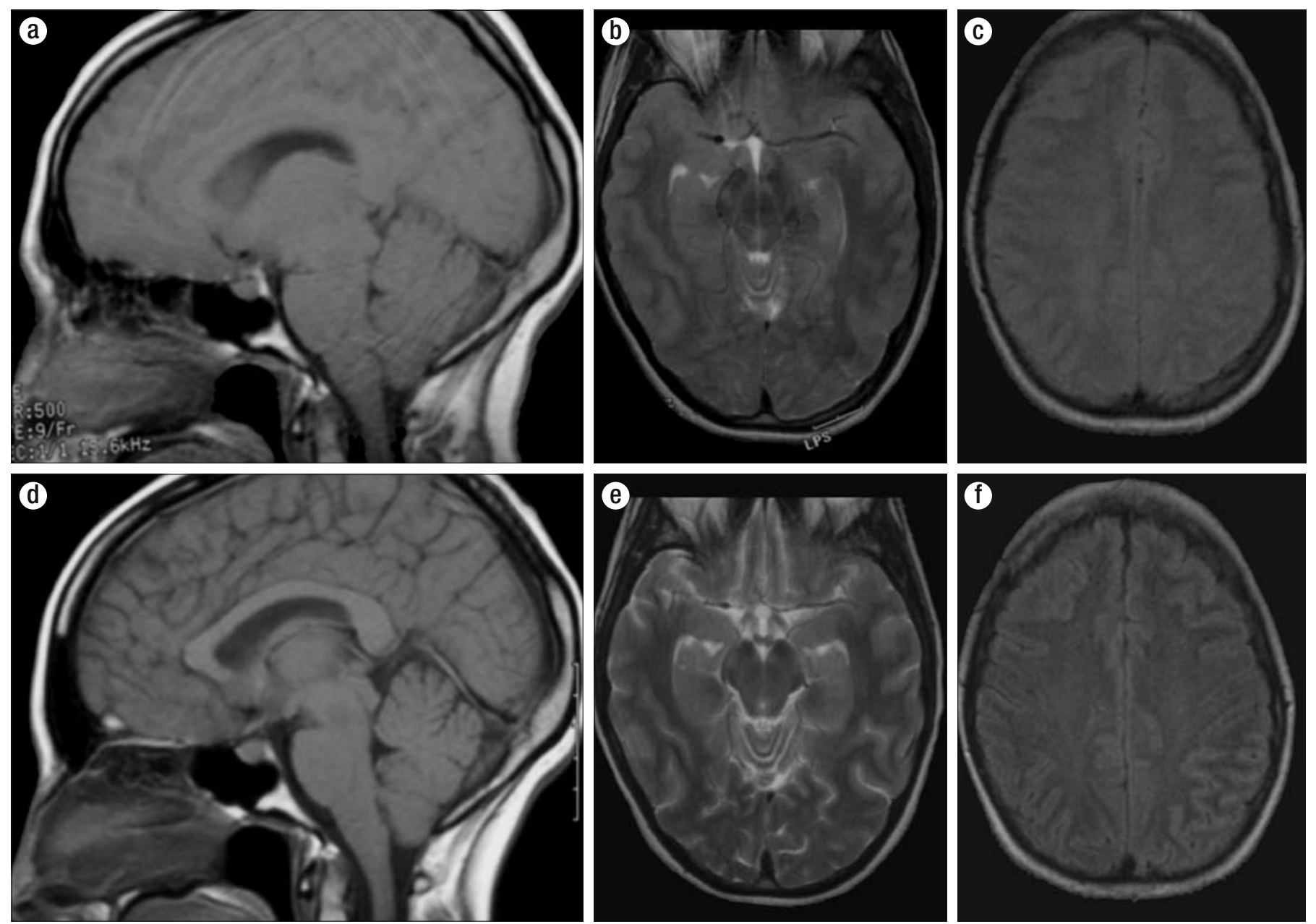

Figure 4. Comparison of MR images during the episode of exercise-associated hyponatremia (top row) and after correction of the hyponatremia (bottom row). (a) Sagittal T1-weighted image upon admission. (b) Axial T2-weighted MR image. (c) Axial fast spin-echo inversion recovery (FSEIR)-weighted image. (d) Sagittal T1-weighted image after correction of hyponatremia. (e) Axial T2-weighted image. (f) Axial FSEIR-weighted image.

DIAGNOSIS: Exercise-associated hyponatremia (EAH) with exercise-associated hyponatremic encephalopathy.

\section{IMAGING FINDINGS}

The imaging findings of hyponatremic encephalopathy relate to the body's attempt to equilibrate the concentration of sodium in the intracellular compartments with that in the extracellular compartments. When the serum sodium rapidly decreases, there is an osmotic shift of fluid into the intracellular spaces. The Monro-Kellie model of intracranial pressure dynamics helps to explain the consequences of this intracellular shift of water with resultant cellular edema and brain swelling. The intracranial contents are grossly made up of brain, cerebrospinal fluid, and blood. An increase in the volume of any one of these components forces a reduction in the volume of the other components. The brain swelling and subsequent reduction of cerebral blood flow account for the clinical symptoms.

The CT and MR images demonstrate an increased volume of brain secondary to cellular swelling, which forces a reduction of the amount of cerebrospinal fluid-manifest as effacement of the cortical sulci and basilar cisterns and a decreased size of the ventricular system. The increased cellular water also leads to a subtle decrease in the cortical density on CT and a lower T1 and higher T2 signal intensity in the cortex on MR imaging. These findings are subtle and diffuse and thus can be difficult to appreciate. A comparison with a normal study in the same patient highlights the changes (Figure 4).

\section{DISCUSSION}

EAH is well known to health care professionals who care for athletes at marathons, triathlons, and other endurance athletic events, since hyponatremia is one of the most common medical complications encountered during and after these events.

A review of the literature shows that most articles concerning EAH have been published within the last 10 years; a large majority of those have been published since 2001. Many articles are case reports such as this. Consensus statements and in-depth review articles are a recent addition to the literature attempting to educate the general medical community about this severe and potentially life-threatening condition. For an excellent in-depth review of the epidemiology, pathogenesis, and therapy of EAH, I would recommend the article by Rosner and Kirven (1).

Until relatively recently, the incidence of hyponatremia during exercise was unknown. A study at the 2002 Boston Marathon found that $13 \%$ of 488 runners studied had hyponatremia 
(2). Another study investigated 330 athletes who finished an ultramarathon race and found that $58 \%$ were hyponatremic (3). Several other studies at endurance events have reported incidence rates up to $29 \%$.

Several risk factors have been linked with EAH. The main risk factors include preexercise overhydration, excessive drinking $(>1.5 \mathrm{~L} / \mathrm{h}$ ) during an athletic event, low body weight, female gender, exercise duration $>4$ hours, use of nonsteroidal antiinflammatory drugs, and a hot environment (4). The study from the 2002 Boston Marathon found that specific predictors for the development of hyponatremia were a fluid intake of $>3 \mathrm{~L}$, a postrace weight greater than prerace weight, water loading prior to the event, and fluid intake during the race (2).

Most of these risk factors were present in our patient. She was female and normally weighed only 104 pounds. Her weight at presentation to the emergency department was 109 pounds. Weight gain during activity is one of the recognized criteria to aid in diagnosis (5). The need for hydration while exercising outdoors had recently been emphasized to her by a physician friend. Following this advice and fearing dehydration and heat exhaustion, she was overhydrating both before and during the workouts. On the day of the current episode, her self-reported total water consumption before, during, and after exercise was $4 \mathrm{~L}$. In addition, she was exercising in the heat and was taking ibuprofen regularly, almost daily, for migraine headaches. The only risk factor that she did not have was exercise duration $>4$ hours.

In addition, the patient's clinical presentation, laboratory findings, imaging findings, and clinical course are classic examples of what one might find with EAH. Interestingly, her clinical symptoms occurred on four consecutive Thursdays following her weekly tennis game and weight training. One week prior to the current admission, she was seen in the Baylor emergency department for a severe headache that was thought to be related to heat exhaustion. At that visit, she had a normal head CT scan and abnormal laboratory results, including a serum sodium of $131 \mathrm{mEq} / \mathrm{L}$ (reference range, 137-145), a serum chloride of 95 $\mathrm{mEq} / \mathrm{L}$ (reference range, 98-109), and a hypochromic, microcytic anemia with a hemoglobin of $11 \mathrm{~g} / \mathrm{dL}$ (reference range, 12-16) and a hematocrit of 31.2\% (reference range, 36-47). Two weeks prior, the patient called an ambulance to her house, complaining of a severe headache and a sense of "feeling like I was going to pass out." Three weeks prior, the patient spent most of the day in bed with a severe headache.

Three of the four times, the patient had a "feeling" that something was seriously wrong. A feeling of "impending doom" has been described as one of the symptoms associated with hyponatremic encephalopathy. In addition to overhydration, additional biochemical features associated with the development of EAH were also manifest in this patient.

\section{Syndrome of inappropriate antidiuretic hormone secretion}

In the study by Noakes et al, hyponatremia did not develop in $70 \%$ of the athletes who overconsumed fluids and had an increase in total body water (6). Also, the maximum excretory capacity of the kidneys $(750-1500 \mathrm{~mL} / \mathrm{h})$ combined with losses from sweat and insensible losses should allow most athletes to consume fluids in excess of $1500 \mathrm{~mL} / \mathrm{h}$. This amount is much more than most athletes would consume during exercise. Siegel et al showed that although avid fluid consumption is clearly a risk factor for $\mathrm{EAH}$, this behavior alone should not produce dilutional hyponatremia without a decrease in urine production (7). Clearly, other factors must contribute to the development of EAH in some athletes.

It has been demonstrated that many cases of EAH fulfill the diagnostic criteria for the syndrome of inappropriate antidiuretic hormone secretion (SIADH). During the evaluation of this patient, several clinicians suspected SIADH based on the presence of hyponatremia and elevated urine sodium levels. Also, at one point the patient was concerned about dehydration because she had not urinated and continued to drink water.

There are several potential pathways for stimulation of antidiuretic hormone (also known as arginine vasopressin, or AVP) in exercising athletes. For example, pain, emotional stresses, and physical stresses have been thought to contribute to the nonosmotic release of AVP. AVP may be stimulated appropriately in the setting of volume depletion. Exposure to heat may also stimulate the secretion of AVP.

Several studies have shown a higher incidence of EAH in women. Two of these studies were from the San Diego Marathon and the New Zealand Ironman triathlon. However, a study from the Boston Marathon, which also found hyponatremia more often in women, showed no statistically significant difference when results were corrected for body mass index, racing time, and weight change, suggesting that body size and duration of exercise may explain the gender differences (2).

Despite this finding, some interesting biochemical differences may place women at greater risk of EAH. First, the gender difference might be explained by an inhibitory effect on $\mathrm{Na}^{+}$$\mathrm{K}^{+}$-ATPase by both estrogen and progesterone. This $\mathrm{Na}^{+}-\mathrm{K}+-$ ATPase has an important function in the extrusion of sodium from cells during the development of hyponatremia. Second, inflammatory cytokines are postulated as contributing factors in the development of SIADH with EAH. One of these inflammatory cytokines, interleukin (IL)-6, may partly explain the female preponderance seen in several studies. Rhabdomyolysis or lesser degrees of muscle injury can occur during exercise with the release of inflammatory cytokines such as IL-6. Mastorakos et al demonstrated that IL- 6 can trigger the release of AVP (8). Also, there is a gender difference in production of IL-6 in response to exercise-induced stress, with women producing significantly higher levels and therefore possibly having a greater risk of EAH (1). Our patient did have abnormal elevation of serum creatine phosphokinase, likely from a degree of muscle injury during exercise. Her admission creatine phosphokinase was $259 \mathrm{U} / \mathrm{L}$ (reference range, 30-135).

\section{Prevention}

Education about the risks of fluid overconsumption is the primary goal for prevention of EAH. Universal guidelines for consumption are difficult if not impossible to develop because of the wide variability in sweat production and renal water 
excretory capacity between individual athletes in the same environmental conditions and in the same individual under different environmental conditions. Generally, it has been suggested that fluid intake should be determined by thirst and that the maximum fluid intake should be between 400 and $800 \mathrm{~mL} / \mathrm{h}$. The casual athlete is much less likely to follow the more detailed guidelines established by USA Track and Field (4). This set of recommendations includes utilizing various methods to estimate hourly sweat losses during exercise and avoiding fluid consumption in excess of this amount during exercise. The plan even includes serial measurements of weight before, during, and after exercise, with the goal being to finish the exercise either at or below the prerace weight.

\section{Therapy}

The treatment of severe or symptomatic EAH requires the administration of hypertonic saline. Although there is no consensus about the amount and concentration of hypertonic saline that can be given safely, the generally recognized therapy in the field is intravenous administration of $100 \mathrm{~mL}$ of $3 \%$ saline over 10 minutes. This infusion will raise the serum sodium concentration 2 to $3 \mathrm{mmol} / \mathrm{L}$ in a short period. Further use of hypertonic saline should be restricted to the hospital setting, where the serum sodium can be closely monitored. In general, $3 \%$ hypertonic saline can be given at 1 to $2 \mathrm{~mL} / \mathrm{kg}$ per hour with close monitoring of serum sodium and urine electrolytes. This rate may need to be increased to 3 to $4 \mathrm{~mL} / \mathrm{kg}$ per hour depending on the severity of the antidiuresis. There have been no reported cases of osmotic demyelination syndrome after the treatment of acute EAH.

\section{CONCLUSION}

EAH is a potentially devastating condition that can occur in otherwise healthy individuals. With the necessary education, which stresses appropriate fluid consumption while exercising, this condition should be easily preventable. Understanding the complex pathophysiology and being able to recognize the signs and symptoms of this condition in the appropriate setting allow early initiation of therapy and an often favorable outcome.

\section{Acknowledgments}

I thank Joshua C. Hammond, who assisted with the digital CT and MR images. The patient is actually my wife, Terry Scott Schucany. I would like to thank her for letting me write up the case and for being the most amazing wife in the world.

1. Rosner MH, Kirven J. Exercise-associated hyponatremia. Clin J Am Soc Nephrol 2007;2(1):151-161.

2. Almond CS, Shin AY, Fortescue EB, Mannix RC, Wypij D, Binstadt BA, Duncan CN, Olson DP, Salerno AE, Newburger JW, Greenes DS. Hyponatremia among runners in the Boston Marathon. $N$ Engl J Med 2005;352(15):1550-1556.

3. Speedy DB, Noakes TD, Rogers IR, Thompson JM, Campbell RG, Kuttner JA, Boswell DR, Wright S, Hamlin M. Hyponatremia in ultradistance triathletes. Med Sci Sports Exerc 1999;31(6):809-815.

4. Casa DJ. Proper hydration for distance running —identifying individual fluid needs. A USA Track \& Field Advisory. Available at http://www.usatf.org/ groups/Coaches/library/hydration/properHydrationForDistanceRunning. pdf; accessed July 24, 2007.

5. National Athletic Trainers' Association. Inter-Association Task Force on Exertional Heat Illnesses Consensus Statement. Available at http://www.nata. org/statements/consensus/heatillness.pdf; accessed July 24, 2007.

6. Noakes TD, Sharwood K, Speedy D, Hew T, Reid S, Dugas J, Almond C, Wharam $\mathrm{P}$, Weschler L. Three independent biological mechanisms cause exercise-associated hyponatremia: evidence from 2,135 weighed competitive athletic performances. Proc Natl Acad Sci US A 2005;102(51):1855018555.

7. Siegel AJ, Verbalis JG, Clement S, Mendelson JH, Mello NK, Adner M, Shirey T, Glowacki J, Lee-Lewandrowski E, Lewandrowski KB. Hyponatremia in marathon runners due to inappropriate arginine vasopressin secretion. Am J Med 2007;120(5):461.e11-e17.

8. Mastorakos G, Weber JS, Magiakou MA, Gunn H, Chrousos GP. Hypothalamic-pituitary-adrenal axis activation and stimulation of systemic vasopressin secretion by recombinant interleukin- 6 in humans: potential implications for the syndrome of inappropriate vasopressin secretion. J Clin Endocrinol Metab 1994;79(4):934-939. 